\title{
Seismic modelling of the $\beta$ Cep star EN (16) Lacertae
}

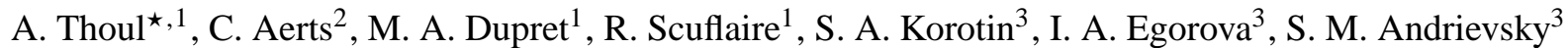 \\ H. Lehmann ${ }^{4}$, M. Briquet ${ }^{1}$, J. De Ridder ${ }^{\star \star, 2}$, and A. Noels ${ }^{1}$ \\ ${ }^{1}$ Institut d'Astrophysique et de Géophysique de Liège, Université de Liège, allée du Six Août 17, 4000 Liège, Belgium \\ 2 Instituut voor Sterrenkunde, Katholieke Universiteit Leuven, Celestijnenlaan 200 B, 3001 Leuven, Belgium \\ 3 Odessa Astronomical Observatory and Isaac Newton Institute of Chile, Odessa Branch, Ukraine \\ 4 Thüringer Landessternwarte, 07778 Tautenburg, Germany
}

Received 12 February 2003 / Accepted 15 May 2003

\begin{abstract}
We perform seismic modelling of the massive $\beta$ Cep star EN Lacertae. The starting point of our analysis is the spectroscopic mode identification recently performed. To this, we add a new updated photometric mode identification based upon a non-adiabatic description of the eigenfunctions in the outer atmosphere. Both mode identifications agree and this allows us to fine-tune the stellar parameters of EN Lacertae with unprecedented precision. This is done by producing a huge amount of stellar models with different parameters and selecting those that fulfill the frequency values and the mode identification. Our study is the first one of its kind in which a reconcilation between observed pulsational characteristics and theoretical models can be achieved at a level that allows accurate determination of the basic stellar parameters of a massive oscillator. We derive a mass of $M=9.62 \pm 0.11 M_{\odot}$ and an age of 15.7 million years if we assume that convective overshooting does not occur.
\end{abstract}

Key words. stars: binaries: spectroscopic - stars: variables: general - stars: individual: EN(16) Lac

\section{Introduction}

The topic of mode identification of non-radial oscillations in main-sequence stars with $\kappa$-driven modes is receiving a lot of attention at the moment (see e.g. Thomson et al. 2003 for the latest summary of efforts). The reason is that a seismic analysis of such stars can only be done successfully once unambiguous mode identification is achieved. One can then use the values of the wavenumbers $(\ell, m)$, together with the frequency values to constrain the star's physical parameters, such as the mass, the luminosity, the effective temperature, the metallicity and the age. Such basic seismic modelling is the subject of our current study.

EN (16) Lacertae (B2IV, hereafter shortened as EN Lac) is a non-radial $\mathrm{p}$-mode oscillator of the class of $\beta$ Cep stars. At the same time, it is also an eclipsing spectroscopic binary, of which the orbital parameters and pulsational frequencies were fine-tuned recently by Lehmann et al. (2000, hereafter called Paper I). For the frequencies of the three modes of the star, we here adopt the latest values derived in Paper I: $f_{1}=5.91128 \mathrm{~cd}^{-1}, f_{2}=5.85290 \mathrm{~cd}^{-1}, f_{3}=5.50259 \mathrm{~cd}^{-1}$.

The investigation of ENLac is important, as it is a prime testcase to perform a ground-based seismic study of a massive star, i.e. a star with a large convective core.

Send offprint requests to: A. Thoul, e-mail: anne.thoul@ulg. ac . be

* Chercheur Qualifié au Fonds National de la Recherche Scientifique, Belgium.

$\star \star$ Postdoctoral Fellow of the Fund for Scientific Research, Flanders.
Dziembowski \& Jerzykiewicz (1996) were the first to attempt a seismic analysis. The latter was hampered, however, by the multitude of possibilities among theoretically predicted modes. Meanwhile, spectroscopic mode identification was done by Aerts et al. (2002, hereafter called Paper II) and it turns out to be consistent with the photometric mode identification (for a summary of the latter so far, we refer to Chapellier et al. 1995). It is then clear that EN Lac is one of the few $\beta$ Cep stars of which we have sufficient consistent information to try a seismic analysis, which is the topic of the current paper.

The paper is organized as follows. We present the derivation of the stellar parameters of the star, including the results of our abundance analysis, in Sect. 2. Further, we perform a new photometric mode identification and discuss its implications for the metallicity of the star in Sect. 3. Section 4 is then devoted to the search for the best stellar models for the star. We end with concluding remarks in Sect. 5.

\section{Stellar parameters}

In order to constrain the stellar models, it is necessary to have a good estimate of the physical parameters of the star. It is well known that the estimate of the effective temperature of $\mathrm{B}$ stars is quite well developed in terms of photometric indices, contrary to the determination of the gravity. Temperature estimates for EN Lac were provided by Jerzykiewicz \& Sterken (1980): $\log T_{\text {eff }}=4.357$ and by Shobbrook (1985): $\log T_{\text {eff }}=$ 4.333. Both these values are based upon Strømgren indices for 
the star. The $\log g$ estimate by Shobbrook amounts to 3.859 . Heynderickx et al. (1994) also provide temperature and gravity estimates, this time based upon Geneva photometry: $\log T_{\text {eff }}=$ 4.352 and $\log g=3.745$. These values, however, are derived from a very limited amount of data and therefore have to be regarded as less reliable.

Very recently, Venn et al. (2002) have made a study of boron abundance of a sample of B-type stars, among which EN Lac, on the basis of HST STIS spectra. In doing so they have derived an effective temperature of $\log T_{\text {eff }}=4.36 \pm 0.02$. Boron is undepleted in EN Lac, suggesting that strong rotationally induced mixing did not (yet) occur during the mainsequence lifetime of the star. Venn et al. have also determined metal abundances for their sample stars and find $[\mathrm{M} / \mathrm{H}]=$ $0.16 \pm 0.14$ for $\mathrm{EN} \mathrm{Lac}$ for $V_{\mathrm{t}}=1 \mathrm{~km} \mathrm{~s}^{-1}$, i.e. $Z \simeq 0.032$.

In view of the large uncertainty for the metal abundance and effective temperature, we have derived elemental abundances from our numerous echelle spectra. The abundances were determined using a plane-parallel atmosphere model (Kurucz 1998 grid). The $\log g$ value needed for this was independently derived by us from a comparison of synthetic and observed profiles of the $\mathrm{H} \beta$ line. The synthetic spectrum codes we used for this are those published by Hubeny et al. (1994) and by Tsymbal (1996) and lead to $\log g=3.80 \pm 0.2$, which is in fact in excellent agreement with the photometric estimates given above. The microturbulent velocity was determined by avoiding any dependence between the iron and carbon abundance from individual lines and their equivalent widths. We found in this way $V_{\mathrm{t}}=3.0 \pm 0.3 \mathrm{~km} \mathrm{~s}^{-1}$. The temperature value resulting from the abundance determination was $\log T_{\text {eff }}=4.36 \pm 0.02$, which is slightly higher but not incompatible with the photometric estimates.

The elemental abundances were derived in LTE approximation using the Kurucz WIDTH9 code. For the elements C, N and O, however, we used a NLTE approximation to derive the abundances. In order to do so, we updated an implementation of the MULTI code (Carlsson 1986). The modification of the code, of the atomic models and of the atomic level characteristics are given in detail in respectively Korotin et al. (1999a, 1999b), Andrievsky et al. (1999) and Korotin et al. (1999c). The results of our abundance analysis are presented in Table 1. We find the star to be very slightly metal poorer than the Sun, and derive $Z \simeq 0.014$.

Adding all these results together, we finally conclude that the range of the stellar parameters of EN Lac to be considered for the modelling is the following: $\log T_{\text {eff }} \in[4.33 ; 4.36]$, $\log g \in[3.7 ; 3.9]$ and $Z \in[0.014 ; 0.030]$.

\section{Photometric mode identification}

Recently, Dupret et al. (2003, hereafter called Paper III) have presented an improved version of the method of photometric mode identification. This improved method is based on the computation of the non-adiabatic eigenfunctions in the entire non-grey atmosphere of the stellar model, according to the formalism outlined by Dupret et al. (2002). The amplitudes and phases of effective temperature and gravity variations can then be determined accurately and used for the photometric mode
Table 1. Results of our abundance analysis of EN (16) Lac. All values are given relative to solar abundances. NL indicates the number of used lines.

\begin{tabular}{rrrrc}
\hline \hline Element & $\mathrm{NL}$ & $(\mathrm{El} / \mathrm{H})$ & {$[\mathrm{El} / \mathrm{H}]$} & $\sigma$ \\
\hline $\mathrm{He}$ & 4 & 11.00 & 0.00 & 0.20 \\
$\mathrm{C}$ & 13 & 8.16 & -0.36 & 0.06 \\
$\mathrm{~N}$ & 18 & 7.64 & -0.28 & 0.09 \\
$\mathrm{O}$ & 11 & 8.48 & -0.35 & 0.08 \\
$\mathrm{Ne}$ & 10 & 8.43 & 0.35 & 0.13 \\
$\mathrm{Mg}$ & 3 & 7.18 & -0.40 & 0.20 \\
$\mathrm{Al}$ & 2 & 6.77 & 0.30 & 0.05 \\
$\mathrm{Si}$ & 6 & 7.00 & -0.55 & 0.25 \\
$\mathrm{~S}$ & 15 & 6.88 & -0.45 & 0.10 \\
$\mathrm{Ar}$ & 1 & 6.55 & 0.15 & - \\
$\mathrm{Fe}$ & 30 & 7.42 & -0.08 & 0.09 \\
\hline
\end{tabular}

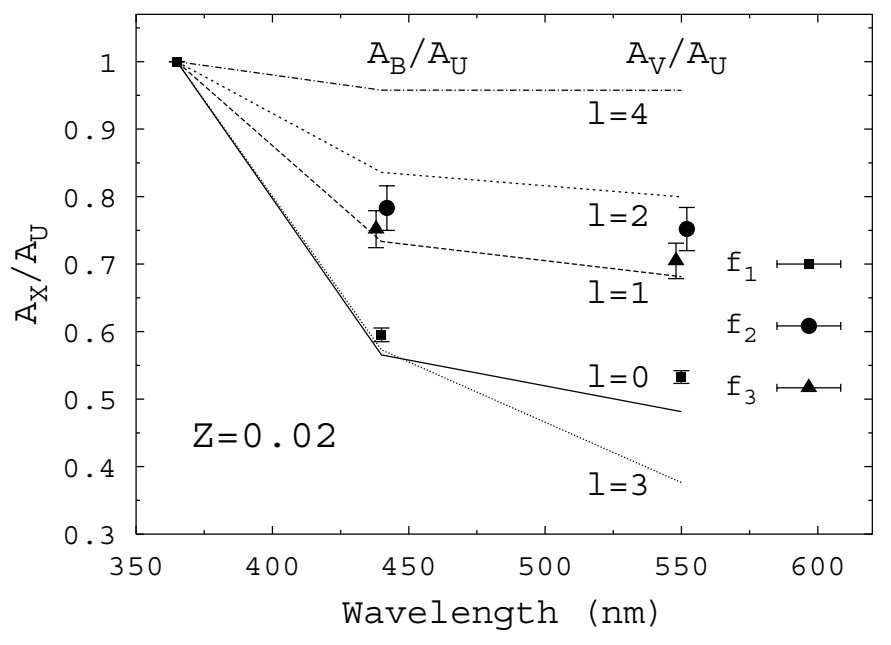

Fig. 1. Observed amplitude ratios with Johnson photometry for the three observed frequencies (error bars) and theoretical predictions for $0 \leq \ell \leq 4$ (lines), for a model with $M=9.7 M_{\odot}, Z=0.02, \log T_{\text {eff }}=$ 4.344 and $\alpha_{\mathrm{ov}}=0$.

identification. In this way, one eliminates two "ad-hoc" parameters used in previous versions of the method.

Given the different results on the degree of the three modes of EN Lac derived from multicolour photometry in the literature (see Chapellier et al. 1995), it is certainly worthwhile to apply Dupret et al.'s new version to EN Lac. We have done so for a set of stellar models with parameters in between those given in Sect. 2, and confronted the theoretical results to the observed amplitude ratios by Jerzykiewicz (1993). For all these models, we find the same most likely identification, which is graphically depicted in Fig. 1 for one of the considered models with solar metallicity. This result of $\ell_{1}=0, \ell_{2}=2, \ell_{3}=1$ was already suggested by Chapellier et al. (1995).

We do note in Fig. 1 that, for the main radial mode, the agreement between the theoretical model (full line) and the observations is not within the observational uncertainty. This conclusion has led Dupret et al. (2003) to further confront the observed photometric amplitude ratios and non-adiabatic 
theoretical predictions by changing the metallicity. Indeed, as shown in Paper III, the theoretical amplitude ratios are very dependent on the metallicity of the model for B-type pulsators. It was shown in Paper III (Fig. 9) that, in the case of EN Lac, a much better agreement between observations and theory could be found for a model slightly less metallic than the Sun: $Z \simeq 0.015$. Moreover it was shown in Paper III that the constraint on the metallicity obtained by this method is not sensitive to the uncertainties on the global stellar parameters $\left(T_{\text {eff }}\right.$, mass, ...). Finally, we note that values below $Z=0.015$ do not lead to excitation of the modes for the stellar parameters appropriate for EN Lac.

We note that we get an ambiguous result for the photometric identification of $f_{2}$, which is very often the case for mode identification from multicolour photometry of $\delta$ Scuti and $\beta$ Cep stars. The difference between the theoretical and observed amplitude ratios can have different origins. Firstly, as said above, the theoretical results are sensitive to the metallicity of the model. For the fundamental radial mode, we obtain a better fit between the theoretical and observed amplitude ratios for a model less metallic than the Sun (see Dupret et al. 2003). Secondly, as shown recently by Daszynska-Daszkiewicz et al. (2002), the coupling between spheroidal modes due to rotation can affect the photometric amplitudes and phases. Thirdly, we note that the observational error bars for the photometric amplitudes of $f_{2}$ are significant. However, the spectroscopic mode identification performed by Aerts et al. (Paper II) leaves no doubt about the $\ell=2, m=0$ nature of the mode with frequency $f_{2}$.

As conclusion for this section, the confrontation between the theoretical and observed amplitude ratios points towards the mode identification $\ell_{1}=0, \ell_{2}=2, \ell_{3}=1$ and a metallicity: $Z \in[0.015 ; 0.020]$.

Our photometric mode identification is fully consistent with the spectroscopic mode identification done recently in Paper II. In Paper II, it was not possible to discriminate between $\ell_{3}=1$ and 2 . The photometric analysis does seem to point out that this second option is less likely. Moreover, we recall that, from the spectroscopic analysis, the second frequency is, without any doubt, identified as an $\ell_{2}=2, m_{2}=0$ mode.

\section{Seismic modelling}

We have a relatively good knowledge of the effective temperature of EN Lac (see discussion in Sect. 2), and, since it is in a binary, we can deduce the value of its luminosity (Pigulski $\&$ Jerzykiewicz 1988). Furthermore, two of its frequencies have been well-identified as being $\ell_{1}=0, m_{1}=0$ and an $\ell_{2}=2, m_{2}=0$ modes, as was shown in Paper II. It therefore seems worthwhile to confront the current models of massive B2IV stars with the constraints put forward by the pulsational character of EN Lac.

We have computed numerous evolutionary sequences from the main sequence with CLÉS (Code Liégeois d'Évolution Stellaire). In doing so we have adopted masses between 8.5 and $10.5 M_{\odot}$, metallicities $Z$ between 0.014 and 0.030 , and two different values of $X, 0.69$ and 0.70 , with and without overshooting $\alpha_{\mathrm{ov}}=0$ or 0.2 . We use the CEFF equation of state

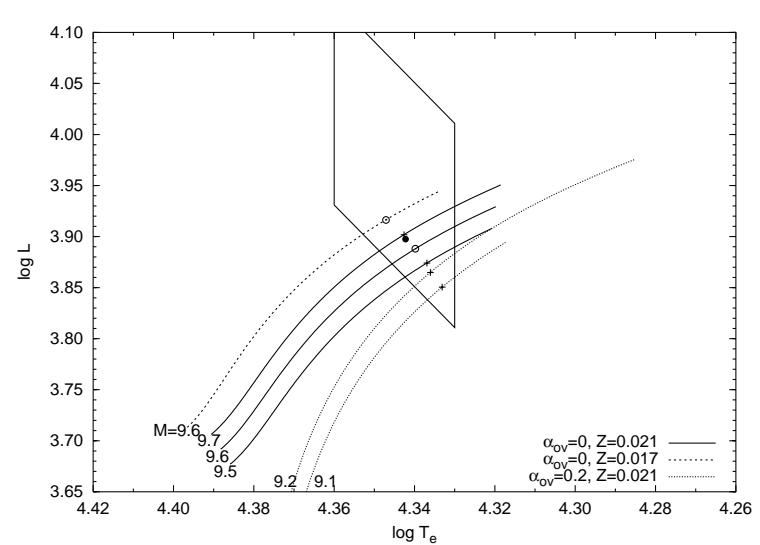

Fig. 2. Evolutionary tracks of stars with masses around $9.5 M_{\odot}$, with $X=0.70$ and for different values of the metallicity $Z$ and the overshooting parameter $\alpha_{\mathrm{ov}}$. The full lines and the dashed line are obtained with models without overshooting, the dotted lines represent evolutionary tracks obtained with $\alpha_{\mathrm{ov}}=0.2$. The error box corresponds to the values for $T_{\text {eff }}$ and $\log g$ given at the end of Sect. 2. The crosses and the dots represent models which present an exact fit for the frequency of the radial fundamental mode $p_{1}$. The black dot corresponds to the model which gives exact fits for the frequencies of the two identified modes $\ell_{1}=0, m_{1}=0$ and $\ell_{2}=2, m_{2}=0$, for a $9.6 M_{\odot}$ star and $\alpha_{\mathrm{ov}}=0$. This model corresponds to $Z=0.0183$.

(Christensen-Dalsgaard \& Däppen 1992). The opacities are those of the Lawrence Livermore National Laboratory (Iglesias \& Rogers 1996) completed with the Alexander \& Ferguson (1994) opacities at low temperature, both tables being smoothly joined in the temperature range defined by $3.95<\log T<4.10$. The nuclear reaction rates are from Caughlan \& Fowler (1988) and the screen factor comes from Salpeter (1954). The boundary conditions at the photosphere are deduced from Kurucz (1998) models. We show examples of the evolutionary tracks we obtained in Fig. 2.

For each model we have calculated the oscillation frequencies using a standard adiabatic code (Boury et al. 1975), and we have selected, for each evolutionary sequence, the model which gives an exact fit for the frequency of the radial fundamental mode $p_{1}\left(\ell_{1}=0, m_{1}=0\right)$. These selected models are shown as crosses and open dots in Fig. 2. We note that selecting a higher order for the mode with frequency $f_{1}$ is not compatible with the other two frequencies and mode identifications. Moreover these models would be much colder than the observed effective temperature.

The second mode has also been well identified as an $\ell_{2}=$ $2, m_{2}=0$ mode. The confrontation to the observed frequency shows without any doubt that it is a $g_{1}$ mode. We have therefore selected, among the models which fit exactly the first frequency, those which also give an exact fit for the second frequency. For each value of the stellar mass $M$, and for fixed $X$ and $\alpha_{\mathrm{ov}}$, this will give us one selected value for the metallicity $Z$, or, alternatively, for each value of $Z$, we get one value of the mass $M$ for which we get exact fits for those two frequencies. The relationship thus obtained between the stellar mass and its metallicity is represented in Fig. 4, for $X=0.70$. We stress here that the mass-metallicity relationship shown in Fig. 4 is not a general $M-Z$ relation, but represents simply the 


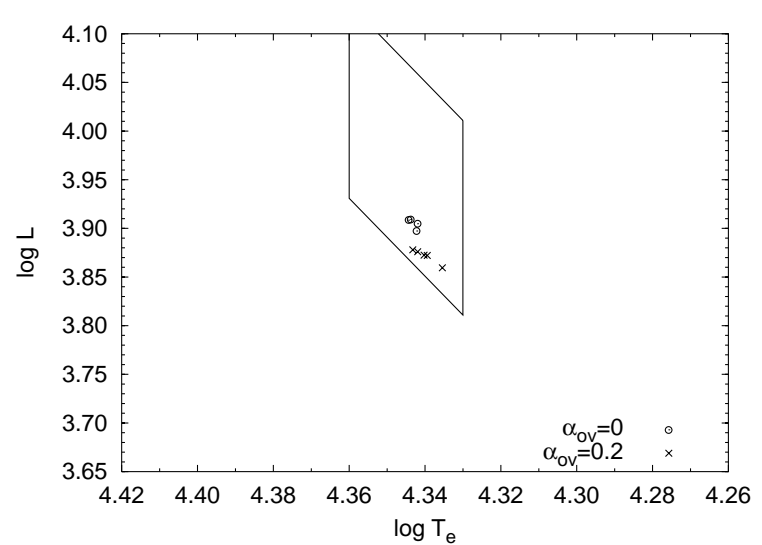

Fig. 3. Positions, in the HR diagram, of the models which fit both the radial fundamental mode $p_{1}\left(l_{1}=0, m_{1}=0\right)$ and the second frequency corresponding to the $g_{1}\left(l_{2}=2, m_{2}=0\right)$ mode.

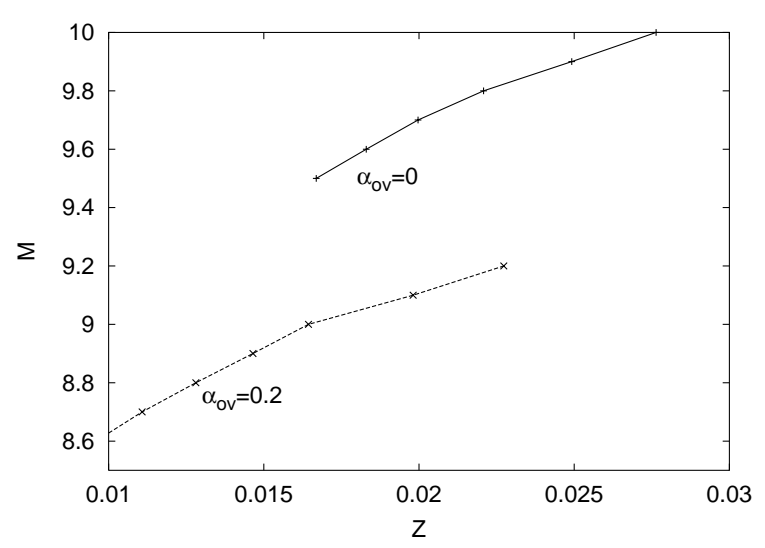

Fig. 4. Relation between the stellar mass (in units of $M_{\odot}$ ) and the metallicity obtained by imposing exact fits for the frequencies of the two identified oscillation modes.

constraint we get on the models for this star by imposing that the models must reproduce the observed frequencies. Changing slightly the value of $X$ is equivalent to changing slightly the value of $Z$. From now on, we will therefore discuss only the results obtained by adopting $X=0.70$. The result obtained for an $M=9.6 M_{\odot}$ star is shown as a black dot in Fig. 2. We see here that the selected model falls very close to the effective temperature as determined by Shobbrook (1985) and by Heynderickx et al. (1994). In fact, all the stellar models which give exact fits for the first two frequencies fall very close to each other in the HR diagram, as shown in Fig. 3. This is especially true for models without overshooting, represented as dots in that figure. We insist here on the fact that the value of the effective temperature has not been imposed in the theoretical models, and is a result of the fitting of the two oscillation frequencies.

Finally, for each model which satisfies exact fits for the frequencies of the two identified oscillation modes, we have computed the frequency of the $\ell=1, g_{1}$ mode. Indeed, we are able to confirm that the third frequency can only be an $\ell_{3}=1$, $g_{1}$ mode, as the frequencies of other modes are too far from the observed value. Therefore, $m_{3}=-1,0$, or +1 .

Using the relation $v_{3}=v_{3,0}+\beta m_{3} v_{\text {rot }}$, where $v_{3}$ is the observed value of the third frequency, $v_{3,0}$ is the theoretical

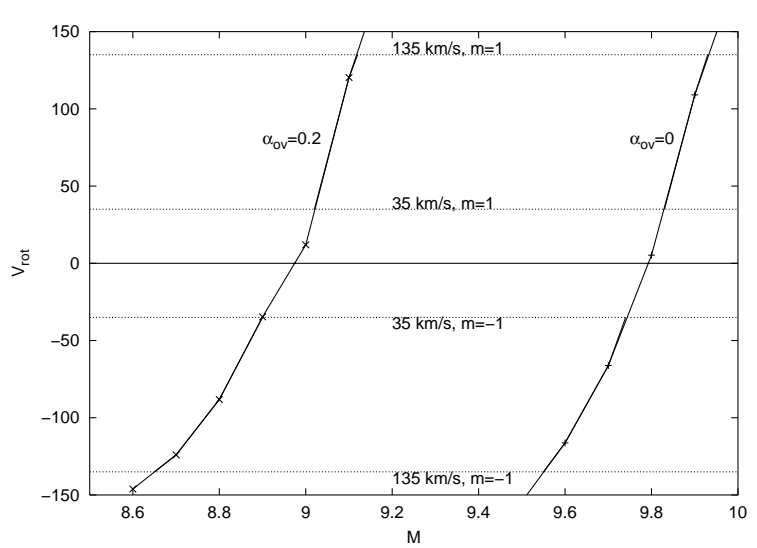

Fig. 5. Rotation velocity obtained for models which fit exactly the two identified modes. The observational constraint $v_{\text {rot }} \in[35,135] \mathrm{km} \mathrm{s}^{-1}$ (see Paper II) is shown as thicker lines.

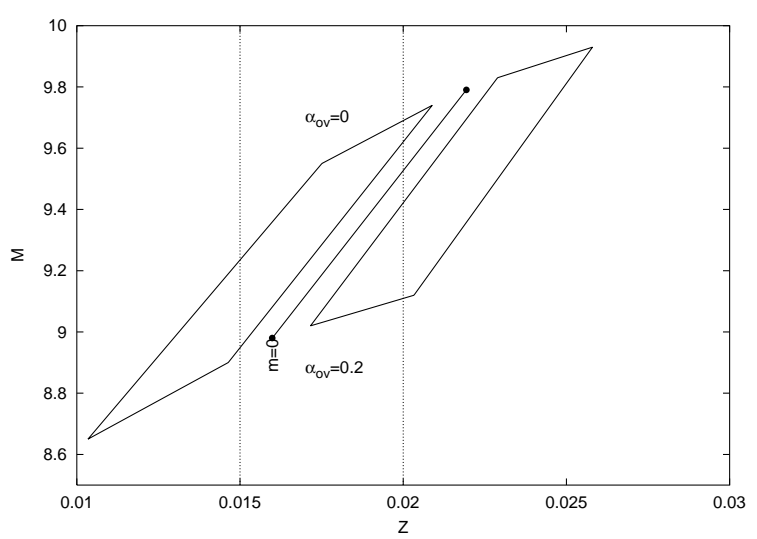

Fig. 6. Allowed domain for the stellar parameters $M$ and $Z$ for the star EN Lac, for $X=0.70$ and for values of $\alpha_{\mathrm{ov}}$ between 0 and 0.2 . The thick lines represent the results obtained for $\alpha_{\mathrm{ov}}=0$ and 0.2 , assuming that $m_{3}= \pm 1$. The black dots represent the results obtained assuming that $m_{3}=0$. The dotted lines represent the constraints on the metallicity obtained through the photometric mode identification (see Sect. 3), $Z \in$ [0.015; 0.020].

value of the frequency of the $\ell_{3}=1$ mode, and $\beta$ is the rotational splitting constant due to rotation, we can obtain for each model the value of the stellar equatorial rotation velocity using $v_{\mathrm{rot}}=2 \pi R v_{\mathrm{rot}}$, where $R$ is the stellar radius.

The results are shown in Fig. 5. We see that the observational constraint $v_{\text {rot }} \in[35,135] \mathrm{km} \mathrm{s}^{-1}$ provided in Paper II delimits acceptable domains for the value of the stellar mass. This domain depends on the value adopted for the overshooting parameter $\alpha_{\mathrm{ov}}$.

This latest constraint can be exported to Fig. 4, to delimit an acceptable area of stellar parameters $M$ and $Z$, as shown in Fig. 6. With the metallicity constraint obtained through the photometric mode identification (see Sect. 2: $Z \in$ $[0.015 ; 0.020]$ ), we obtain that the mass of $16 \mathrm{Lac}$ is between 9 and $9.7 M_{\odot}$. We note that the major uncertainty on the stellar mass comes from the unknown value of the overshooting parameter $\alpha_{\mathrm{ov}}$. If we assume that $\alpha_{\mathrm{ov}}=0$, the constraint on the mass is much narrower; we get $M \in[9.55,9.70]$, corresponding to $Z \in[0.017,0.020]$. In this case, we also have very precise values for the effective temperature and the luminosity, as seen 


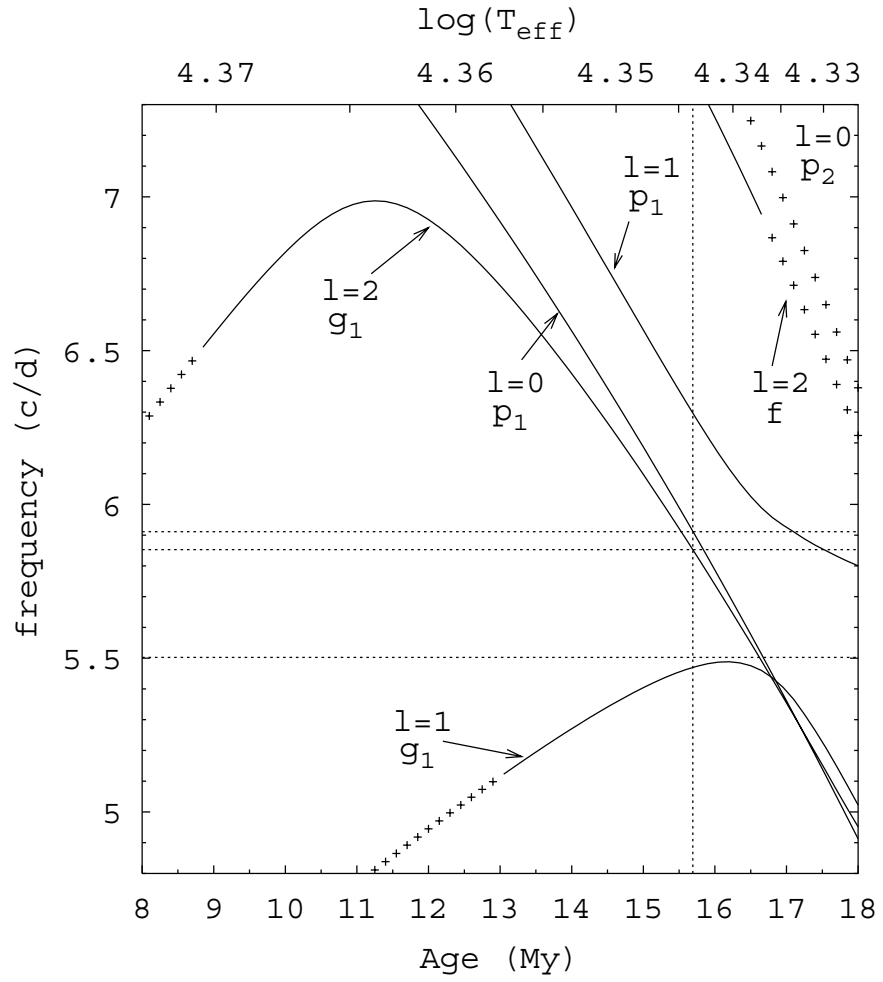

Fig. 7. Evolution of the frequencies as a function of age (Myear) for models with $M=9.79 M_{\odot}, Z=0.02193$ and $\alpha_{\text {ov }}=0$. The three horizontal lines correspond to the three observed frequencies of EN Lac. The vertical line corresponds to the model giving the best fit between the theoretical and observed frequencies. The full lines correspond to the unstable modes and the " + " to the stable modes.

in Fig. 3: $\log T_{\text {eff }}=4.34$ and $\log L / L_{\odot}=3.90$. These values are in the observational error box.

Finally, we give in Fig. 7 a general view of the evolution of the frequencies as a function of age around one of our best models: $M=9.79 M_{\odot}, Z=0.02193$ and $\alpha_{\text {ov }}=0$. We see that the model with age $=15.69 \mathrm{My}$ and $\log T_{\text {eff }}=4.3438$ (vertical line) fits very well the three observed frequencies. We note that the mode $\ell=1, g_{1}$ is just undergoing an avoided crossing at this evolution stage. In this figure, the solid lines correspond to the unstable modes and the "+" to the stable modes as predicted by our non-adiabatic code.

\section{Conclusions}

Three frequencies have recently been obtained with very high precision from the observed radial-velocity variations for the star EN Lac (Paper I), and the spectroscopic mode identification has been performed (Paper II). In this paper, we present the results of the photometric mode identification, performed using the improved method of Dupret et al. (2003). The photometric mode identification confirms the results obtained before, but it also reduces considerably the uncertainty on the star's metallicity. Evolutionary tracks and oscillation frequencies were calculated for the star, for different values of the mass, the metallicity, and the overshooting parameter. By imposing exact fits with the observed frequencies of the two wellidentified modes, we get a relationship between the mass and the metallicity, for a given value of $\alpha_{\mathrm{ov}}$. The observational constraints on the equatorial rotation velocity further reduces the allowed range for the stellar mass, or, equivalently, for the metallicity. We have obtained the following results for the parameters of EN Lac: $M \in$ [9.0; 9.7] $M_{\odot}, Z \in[0.015$; 0.020], and $\left|v_{\text {rot }}\right| \in[0.11,0.44] \mathrm{c} / \mathrm{d}$. The major uncertainty comes from the value adopted for the overshooting parameter.

The current view in constructing evolutionary models of massive stars is that overshooting is not a necessary ingredient as it is not needed to explain the observational pulsational properties of a large unbiased sample of such stars (see, e.g., Pamyatnykh 1999). In that case, i.e. if we assume that $\alpha_{\mathrm{ov}}=0$, the mass and the metallicity of EN Lac are much better determined from our study. We get $M \in[9.55 ; 9.70] M_{\odot}$ and $Z \in[0.017 ; 0.020]$. A mass-determination with an uncertainty below $2 \%$ is unique for such a massive star, which points immediately to the power of asteroseismology for such objects. The uncertainty on the stellar parameters now comes only from the uncertainty in the equatorial rotation velocity.

Finally, by fitting the frequencies we have also obtained very precise values for the effective temperature, luminosity and age of the star. In our analyses we did not have the need to use the observational values for these parameters, but we rather have determined them seismically, with a much higher precision than current direct observations can provide for such a massive star as EN Lac.

Our stellar models are of course dependent on the physics included in these models (equation of state, nuclear reactions rates, opacities, treatment of the convective core, detailed chemical composition, ...) as well as on the global stellar parameters used (mass, metallicity, effective temperature, luminosity). We have shown in our work that we find complete agreement between the models and the mode identification of the observed frequencies, which is usually not the case for $\kappa$-driven modes in main-sequence stars. A true seismic study ought to provide constraints on the physical description of the star. Until now, however, this is only possible for the Sun, for which thousands of oscillation frequencies have been measured with very high precision.

The situation for other stars is very different from the one in the Sun. $16 \mathrm{Lac}$ is the first $\beta$ Cephei star for which the photometric and spectroscopic identifications of several oscillation frequencies are compatible with each other. Moreover, two of these frequencies have been successfully identified as axisymmetric modes and so are unaffected by rotational splitting. By imposing that our stellar models must reproduce those two frequencies, we have been able to put severe constraints on some of the global parameters of the $\operatorname{star}(M$ and $Z)$. As a by-product of our seismic analysis, we also get values for the luminosity and the effective temperature, which are completely consistent with the observations. It is noteworthy that the derivation of global parameters of massive main-sequence stars is very difficult, as their parallaxes are badly determined. Our seismic estimates of these parameters are of unforeseen accuracy.

Because we only have two identified modes, we cannot, of course, constrain the models further. For example, we would need at least a third well-identified frequency to constrain the overshooting parameter. Our study is a first step towards such 
more in-depth seismic studies of $\beta$ Cephei stars. In the future, we plan to observe more frequencies, to identify them, and with the need to fit all the frequencies with a stellar model, we will then be able to gain more information on the stellar physics inside those stars.

Our study is essentially based on only two frequency values belonging to well-identified axisymmetric modes. Uncertainties in the mode identification have hampered previous seismic studies of main-sequence stars. We note that the problem of mode identification is much easier to solve in the $\beta$ Cep stars than in any other type of main-sequence oscillators with $\kappa$-driven modes as their frequency spectrum is not very dense in the range where oscillations are observed. It is therefore clear that studies analogous to the one presented here will be done in the near future for other $\beta$ Cep stars, and that they will allow in-depth derivations of the internal structure parameters, even with a relatively limited amount of detected and wellidentified modes. A major effort to achieve this from groundbased data from a huge multisite campaign is currently being undertaken by Handler \& Aerts (2002) for the $\beta$ Cep star $v$ Eri. We propose that $\beta$ Cep stars are included as prime targets for the future asteroseismic missions such as MOST, COROT and Eddington.

Acknowledgements. We thank J. Christensen-Dalsgaard who kindly supplied us with the code for the computation of the CEFF equation of state. This work has been supported by the PRODEXESA/Contract\#15448/01/NL/SFe(IC), by the Pole d'Attraction Interuniversitaire Contract \# P5/36, and from FRIA (Belgium).

\section{References}

Aerts, C., Lehmann, H., Briquet, M., et al. 2003, A\&A, 399, 639 (Paper II)

Alexander, D. R., \& Ferguson, J. W. 1994, ApJ, 437, 879

Andrievsky, S. M., Korotin, S. A., Luck, R. E., \& Kostynchuk, L. Yu. 1999, A\&A, 342, 756

Boury, A., Gabriel, M., Noels, A., Scuflaire, R., \& Ledoux, P. 1975, A\&A, 41, 279
Carlsson, M. 1986, Uppsala Obs. Rep. 33

Caughlan, G. R., \& Fowler, W. A. 1988, Atomic data and nuclear data tables, 40, 283

Chapellier, E., Lecontel, \& J. M., Lecontel, D. 1995, A\&A, 304, 406

Christensen-Dalsgaard, J., \& Däppen, W. 1992, A\&A, 4, 267

Daszynska-Daszkiewicz, J., Dziembowski, W. A., Pamyatnykh, A. A., \& Goupil, M.-J. 2002, A\&A, 392, 151

Dupret, M.-A., De Ridder, J., Neuforge, C., Aerts, C., \& Scuflaire, R. 2002, A\&A, 385, 563

Dupret, M.-A., De Ridder, J., De Cat, P., et al. 2003, A\&A, 398, 677 (Paper III)

Dziembowski, W. A., \& Jerzykiewicz, M. 1996, A\&A, 306, 436

Handler, G., \& Aerts, C. 2002, Comm. in Asteroseismology, 142, 20

Heynderickx, D., Waelkens, C., \& Smeyers, P. 1994, A\&AS, 105, 447

Hubeny, I., Lanz, T., \& Jeffery, C. S. 1994, Newslett. Analysis Astron. Spectra, 20, 30

Iglesias, C. A., \& Rogers, F. J. 1996, ApJ, 464, 943

Jerzykiewicz, M. 1993, Acta Astron., 43, 13

Jerzykiewicz, M., \& Sterken, C. 1980, in Variability in stars and galaxies, Fifth European Regional meeting in Astronomy, ed. P. Ledoux (Institut d'Astrophysique de Liège), p. B.4.1

Korotin, S. A., Andrievsky, S. M., \& Kostynchuk, L. Yu. 1999a, ApSS, 260, 531

Korotin, S. A., Andrievsky, S. M., \& Kostynchuk, L. Yu. 1999b, A\&A, 342,756

Korotin, S. A., Andrievsky, S. M., \& Luck, R. E. 1999c, A\&A, 351, 168

Kurucz, R. L. 1998, http://cfaku5 .harvard.edu/grids.html

Lehmann, H., Harmanec, P., Aerts, C., et al. 2001, A\&A, 367, 236 (Paper I)

Pamyatnykh, A. A. 1999, Acta Astron., 49, 119

Pigulski, A., \& Jerzykiewicz, M. 1988, Acta Astron., 38, 401

Salpeter, E. E. 1954, Austral. J. Phys., 7, 373

Shobbrook, R. R. 1985, MNRAS, 214, 33

Thompson, M. J., Cunha, M. S., \& Monteiro, M. J. P. F. G. (eds.) 2003, Asteroseismology Across the HR diagram (Kluwer Academic Publishers), Astrophys. Space Sci. 284

Tsymbal, V. 1996, Model Atmospheres and Spectrum Synthesis, ed. S. J. Adelman, F. Kupka, \& W. W. Weiss, ASP Conf. Ser., 108, 198

Venn, K. A., Brooks, A. M., Lambert, David, L., et al. 2002, ApJ, 565, 571 